\title{
The Mixed Type Splitting Methods for Solving Fuzzy Linear Systems
}

\author{
H. Saberi Najafi, ${ }^{1}$ S. A. Edalatpanah, ${ }^{1}$ and S. Shahabi ${ }^{2}$ \\ ${ }^{1}$ Department of Mathematics, Faculty of Sciences, University of Guilan, University Campus 2, Rasht, Iran \\ ${ }^{2}$ Department of Mathematics, Faculty of Sciences, Islamic Azad University, Tonekabon Branch, Tonekabon, Iran
}

Correspondence should be addressed to S. A. Edalatpanah; saedalatpanah@gmail.com

Received 18 June 2014; Revised 21 October 2014; Accepted 21 October 2014; Published 18 November 2014

Academic Editor: D. Nagesh Kumar

Copyright (c) $2014 \mathrm{H}$. Saberi Najafi et al. This is an open access article distributed under the Creative Commons Attribution License, which permits unrestricted use, distribution, and reproduction in any medium, provided the original work is properly cited.

\begin{abstract}
We consider a class of fuzzy linear systems (FLS) and demonstrate some of the existing methods using the embedding approach for calculating the solution. The main aim in this paper is to design a class of mixed type splitting iterative methods for solving FLS. Furthermore, convergence analysis of the method is proved. Numerical example is illustrated to show the applicability of the methods and to show the efficiency of proposed algorithm.
\end{abstract}

\section{Introduction}

Solving fuzzy systems has been considered by many researchers, for example, [1-9] and the references therein. In $[1,2]$ Kandel et al. applied the embedding method for fuzzy linear system (hereafter denoted by FLS) and replaced the FLS by a $2 n \times 2 n$ crisp linear system. This model has been modified later by some other researchers; see [10-16] and the references therein.

Here, based on mixed type splitting, we introduce a new iterative method to FLS. The mixed type splitting iterative method $[17,18]$ is given for the linear system of equations $A x=b$, where $A$ is positive real. Cheng et al. in [19] presented a class of the mixed type splitting iterative methods based on $[17,18]$ and some convergence conditions were given. They also proposed some sufficient and necessary conditions of convergence when coefficient matrix of the linear system is certain matrices such as $M$-matrix.

In this paper, the mixed type splitting iterative method for FLS will be established, which is a generalization of mixed type splitting iterative method for linear system. Some sufficient conditions for convergence of the mixed type splitting iterative method will be considered. Moreover, we will discuss a comparison theorem, which describes the influences of the parameters on the convergence rates of the new methods.

\section{Preliminaries}

In this section we provide some basic notations and definitions of fuzzy number and fuzzy linear system.

Definition 1. An arbitrary fuzzy number is represented, in parametric form, by an ordered pair of functions $(\underline{u}(r), \bar{u}(r))$, $0 \leq r \leq 1$, which satisfy the following requirements (see $[2,3])$.

(i) $\underline{u}(r)$ is a bounded monotonic increasing left continuous function over $[0,1]$.

(ii) $\bar{u}(r)$ is a bounded monotonic decreasing left continuous function over $[0,1]$.

(iii) $\underline{u}(r) \leq \bar{u}(r), 0 \leq r \leq 1$.

A crisp number $\alpha$ can be simply expressed as $u(r)=\bar{u}(r)=$ $\alpha, 0 \leq r \leq 1$. The addition and scalar multiplication of fuzzy numbers $x=(\underline{x}(r), \bar{x}(r))$ and $y=(y(r), \bar{y}(r))$ can be described as follows:

(i) $x=y$ if and only if $\underline{x}(r)=y(r)$ and $\bar{x}(r)=\bar{y}(r)$;

(ii) $x+y=(\underline{x}(r)+\underline{y}(r), \bar{x}(r)+\bar{y}(r))$;

(iii) for all $K \in R ; K x=\left\{\begin{array}{l}(K \underline{x}, K \bar{x}), K \geq 0, \\ (K \bar{x}, K x), K<0 .\end{array}\right.$ 
Definition 2. Consider the $n \times n$ linear system of the following equations:

$$
\begin{gathered}
a_{11} x_{1}+a_{12} x_{2}+\cdots+a_{1 n} x_{n}=b_{1}, \\
a_{21} x_{1}+a_{22} x_{2}+\cdots+a_{2 n} x_{n}=b_{2}, \\
\vdots \\
a_{n 1} x_{1}+a_{n 2} x_{2}+\cdots+a_{n n} x_{n}=b_{n},
\end{gathered}
$$

where the coefficient matrix $A=\left(a_{i j}\right), 1 \leq i, j \leq n$, is a crisp matrix and $b_{i} \in E^{1}, 1 \leq i \leq n$, is called a FLS.

Definition 3. A fuzzy number vector $X=\left(x_{1}, x_{2}, \ldots, x_{n}\right)^{T}$, given by the parametric form $x_{i}=\left(x_{i}(r), \overline{x_{i}}(r)\right), 1 \leq i \leq n$, $0 \leq r \leq 1$, is called a solution of the FLS (1) if

$$
\begin{aligned}
& \underline{\sum_{j=1}^{n} a_{i j} x_{j}}=\sum_{j=1}^{n} \underline{a_{i j} x_{j}}=\underline{b_{i}}, \\
& \overline{\sum_{j=1}^{n} a_{i j} x_{j}}=\sum_{j=1}^{n} \overline{a_{i j} x_{j}}=\overline{b_{i}} .
\end{aligned}
$$

Friedman et al. $[2,3]$, in order to solve the system given by (2), have solved a $2 n \times 2 n$ crisp linear system as follows:

$$
S X=B \text {, }
$$

where $S=\left(s_{i j}\right)$ are determined as follows:

$$
\begin{gathered}
a_{i j} \geq 0 \longrightarrow s_{i j}=a_{i j}, \quad s_{i+n, j+n}=a_{i j}, \\
a_{i j}<0 \longrightarrow s_{i, j+n}=-a_{i j}, \quad s_{i+n, j}=-a_{i j},
\end{gathered}
$$

and any $\left(s_{i j}\right)$ which is not determined by $(4)$ is zero.

Then referring to $[2,3]$ we have

$$
\begin{aligned}
S & =\left[\begin{array}{ll}
s_{1} & s_{2} \\
s_{2} & s_{1}
\end{array}\right], \quad X=\left[\frac{x}{-\bar{x}}\right], \quad B=\left[\begin{array}{c}
\underline{b} \\
-\bar{b}
\end{array}\right] \\
& \Longrightarrow\left\{\begin{array}{c}
s_{1} \underline{x}-s_{2} \bar{x}=\underline{b}, \\
s_{2} \underline{x}-s_{1} \bar{x}=-\bar{b} .
\end{array}\right.
\end{aligned}
$$

Or

$$
\begin{aligned}
S & =\left[\begin{array}{cc}
s_{1} & -s_{2} \\
-s_{2} & s_{1}
\end{array}\right], \quad X=\left[\frac{x}{\bar{x}}\right], \quad B=\left[\frac{b}{\bar{b}}\right] \\
& \Longrightarrow\left\{\begin{array}{c}
s_{1} \underline{x}-s_{2} \bar{x}=\underline{b}, \\
-s_{2} \underline{x}+s_{1} \bar{x}=\bar{b},
\end{array}\right.
\end{aligned}
$$

where $s_{1}, s_{2} \geq 0$ and $A=s_{1}-s_{2}$.

Definition 4 (see [20]). (a) A matrix $A=a_{i j}$ is called a $Z$ matrix if, for any $i \neq j, a_{i j} \leq 0$.

(b) A $Z$-matrix is an $L$-matrix, if $a_{i i}>0$.

(c) A $Z$-matrix is an $M$-matrix, if $A$ is nonsingular and $A^{-1} \geq 0$. (d) For any matrix $A$ the comparison matrix $\langle A\rangle=$ $\left(m_{i j}\right) \in R^{n \times n}$ is defined by

$$
m_{i i}=\left|a_{i i}\right|, \quad m_{i j}=-\left|a_{i j}\right|, \quad i \neq j, 1 \leq i, j \leq n .
$$

(e) A complex matrix $A$ is an $H$-matrix if $\langle A\rangle=\left(m_{i j}\right) \epsilon$ $R^{n \times n}$ is an $M$-matrix.

(f) Matrix $A$ is called a Generalized Diagonally Dominant Matrix (GDDM) if there exists a positive diagonal matrix $W$ such that $A W$ is a Strictly Diagonally Dominant Matrix (SDDM).

Lemma 5 (see [19]). Let $A$ be the coefficient matrix of the linear system $A x=b$. If $A$ is an $M$-matrix and conditions of (19) are satisfied, then the mixed type splitting iterative method is convergent.

Remark 6. For any splitting, $A=M-N$, where $M$ is nonsingular, the iterative method for solving linear systems of $A x=b$ is

$$
x^{(i+1)}=M^{-1} N x^{(i)}+M^{-1} b, \quad i=0,1, \ldots
$$

This iterative process converges to the unique solution $x=$ $A^{-1} b$ for initial vector value $x^{0} \in R^{n}$ if and only if the spectral radius $\rho\left(M^{-1} N\right)<1$, where $T=M^{-1} N$ is called the iteration matrix [20]. For example, $\operatorname{suppose} \operatorname{diag}(A)=I$ and $A=I-$ $L-U$, where $L$ and $U$ are strictly lower and strictly upper triangular part of $A$, respectively. Then for classical AOR (see; [10]) we have

$$
\begin{aligned}
M_{r, w} & =\frac{1}{w}(I-r L), \\
N_{r, w} & =\frac{1}{w}((1-w) I+(w-r) L+w U) \\
& \Longrightarrow l_{r, w}=M_{r, w}^{-1} N_{r, w} \\
& =(I-r L)^{-1}((1-w) I+(w-r) L+w U) .
\end{aligned}
$$

And when $w=r$, we have SOR method [20]; that is,

$$
\begin{gathered}
M_{w}=\frac{1}{w}(I-w L), \quad N_{w} \\
=\frac{1}{w}((1-w) I+w U) \\
\Longrightarrow l_{w}=M_{w}^{-1} N_{w} .
\end{gathered}
$$

Lemma 7 (see [19]). Let $A$ be the coefficient matrix of the linear system $A x=b$. If $A$ is an L-matrix, conditions of (19) are satisfied and

$$
\begin{aligned}
& 0 \leq D_{d} \leq \frac{1}{w}(1-w) D, \\
& 0 \leq L_{l} \leq\left(1-\frac{r}{w}\right), \\
& 0 \leq r \leq w \leq 1 .
\end{aligned}
$$

Then

(i) If $\rho\left(l_{r, w}\right)<1 \Rightarrow \rho(T) \leq \rho\left(L_{r \cdot w}\right)$. 
(ii) If $\rho\left(l_{r, w}\right)=1 \Rightarrow \rho(T)=\rho\left(L_{r \cdot w}\right)$.

(iii) If $\rho\left(l_{r, w}\right)>1 \Rightarrow \rho(T) \geq \rho\left(L_{r \cdot w}\right)$.

Lemma 8 (see [20]). Matrix $A$ is a GDDM matrix if and only if $A$ is an $H$-matrix.

\section{The Mixed Type Splitting Iterative Methods for Fuzzy Linear Systems}

Let $S$ be nonsingular and $S=D-L-U$, where

$$
\begin{gathered}
{\left[\begin{array}{cc}
s_{1} & -s_{2} \\
-s_{2} & s_{1}
\end{array}\right]\left[\frac{x}{\bar{x}}\right]=\left[\frac{b}{\bar{b}}\right],} \\
D=\left[\begin{array}{cc}
D_{1} & 0 \\
0 & D_{1}
\end{array}\right], \quad L=\left[\begin{array}{cc}
L_{1} & 0 \\
s_{2} & L_{1}
\end{array}\right], \\
U=\left[\begin{array}{cc}
U_{1} & s_{2} \\
0 & U_{1}
\end{array}\right], \quad D_{1}=\operatorname{diag}\left(s_{1}\right)>0, \\
D_{1}-L_{1}-U_{1}=S_{1},
\end{gathered}
$$

and $-L_{1},-U_{1}$ are strictly lower and upper triangular matrices of $s_{1}$, respectively. The iterative method for $S X=B$ is

$$
X^{(i+1)}=M^{-1} N X^{(i)}+M^{-1} B, \quad i=0,1, \ldots,
$$

where $S=M-N$, det $(M) \neq 0$, and $X^{(0)}$ is any initial vector. There are several well-known point iterative methods and block numerical iterative methods for FLS such as Jacobi, Gauss-Seidel, SOR, and AOR; see [9-14]. As a matter of fact, these methods are generalization of iterative methods for crisp linear systems $A x=b$. For instance, in AOR method for FLS [12] we have

$$
x^{(i+1)}=T_{r, w} x^{(i)}+(D-r L)^{-1} w b, \quad i=0,1, \ldots,
$$

where the iterative matrix is

$$
T_{r, w}=(D-r L)^{-1}[(1-w) D+(w-r) L+w U] .
$$

Therefore, we obtain

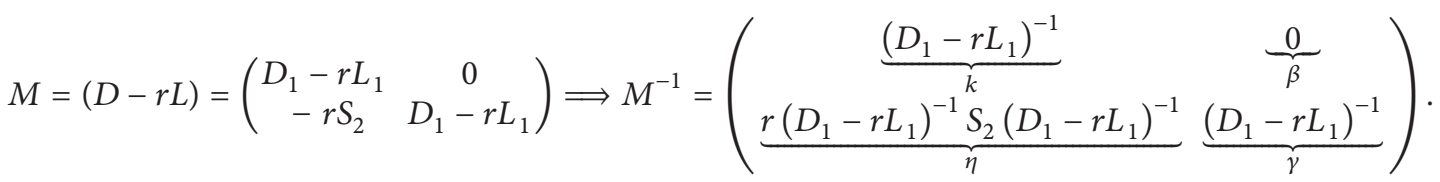

And we have

$$
\begin{aligned}
& T_{r, w}=M^{-1} N=\left(\begin{array}{cc}
k & 0 \\
r k S_{2} k & k
\end{array}\right)\left\{\left(\begin{array}{cc}
(1-w) D_{1} & 0 \\
0 & (1-w) D_{1}
\end{array}\right)+\left(\begin{array}{cc}
(w-r) L_{1} & 0 \\
(w-r) S_{2} & (w-r) L_{1}
\end{array}\right)+\left(\begin{array}{cc}
w U_{1} & w S_{2} \\
0 & w U_{1}
\end{array}\right)\right\} \\
& \Longrightarrow T_{r, w}=\left[\begin{array}{cc}
\underbrace{k\left[(1-w) D_{1}+(w-r) L_{1}+w U_{1}\right]}_{\Phi} & \underbrace{w k S_{2}}_{\Gamma} \\
\underbrace{w k S_{2} k\left[(1-r) D_{1}+r U_{1}\right]}_{\Omega} & \underbrace{k\left[(1-w) D_{1}+(w-r) L_{1}+r w S_{2} k S_{2}+w U_{1}\right]}_{\Psi}
\end{array}\right] .
\end{aligned}
$$

Other methods are the same and we know that, by choosing special parameters, the similar results can be obtained, for example,

(1) Jacobi method for $w=1, r=0$;

(2) JOR (Jacobi Overrelaxation) method for $r=0$;

(3) Gauss-Seidel method for $r=w=1$;

(4) SOR method for $r=w$.

Now, from $X^{(i)}=\left[\frac{x^{(i)}}{\bar{x}^{(i)}}\right]$ we have the following algorithms (mentioned in [12] for the first time).
Algorithm 9. AOR iterative method for FLS.

Step 1. Choose an initial vector $X^{(0)}=\left[\frac{x^{(0)}}{\bar{x}^{(0)}}\right]$ and parameters $r$ and $w$.

Step 2. For $k=0,1,2, \ldots$ do

$$
\begin{aligned}
& \underline{x}^{(i+1)}=\Phi \underline{x}^{(i)}+\Gamma \bar{x}^{(i)}+w(k \underline{b}+\beta \bar{b}) \\
& \bar{x}^{(i+1)}=\Psi \bar{x}^{(i)}+\Omega \underline{x}^{(i)}+w(\eta \underline{b}+\gamma \bar{b}) .
\end{aligned}
$$

Step 3. If $\left\|\bar{x}^{(i+1)}-\bar{x}^{(i)}\right\| /\left\|\bar{x}^{(i+1)}\right\|<\varepsilon$ or $\left\|x^{(i+1)}-x^{(i)}\right\| /\left\|x^{(i+1)}\right\|<$ $\varepsilon$, then stop; otherwise set $k=k+1$ and go to Step 2 . 
Step 4. End for.

Step 5. Return to $X^{(k+1)}$.

Next, we will establish the mixed type splitting iterative method for FLS. The mixed type splitting iterative methods [19] for solving $A x=b$ are given by the following:

$$
\begin{array}{r}
\left(D+D_{d}+L_{s}-L\right) x^{(i+1)}=\left(D_{d}+L_{s}+U\right) x^{(i)}+b, \\
i=0,1,2, \ldots ;
\end{array}
$$

whose iteration matrix is

$$
\begin{aligned}
& T=\left(D+D_{d}+L_{s}-L\right)^{-1}\left(D_{d}+L_{s}+U\right), \\
& A=D-L-U, \quad D_{d} \geq 0, \quad 0 \leq L_{s} \leq L .
\end{aligned}
$$

Now, we consider the mixed type splitting iterative methods for solving FLS. Based on above demonstration, we have

$$
\begin{aligned}
& \underbrace{\left[\begin{array}{cc}
\underbrace{D_{1}+D_{d}}_{E}+L_{s}-L_{1} & 0 \\
L_{2 s}-s_{2} & D_{1}+D_{d}+L_{s}-L_{1}
\end{array}\right]}_{M}\left[\begin{array}{l}
\underline{x}^{(i+1)} \\
\bar{x}^{(i+1)}
\end{array}\right] \\
& =\underbrace{\left[\begin{array}{cc}
D_{d}+L_{s}+U_{1} & s_{2} \\
L_{2 s} & D_{d}+L_{s}+U_{1}
\end{array}\right]}_{N}\left[\frac{\underline{x}^{(i)}}{\bar{x}^{(i)}}\right]]+\left[\begin{array}{l}
\bar{b} \\
\bar{b}
\end{array}\right],
\end{aligned}
$$

where $0 \leq L_{s} \leq L$ and $0 \leq L_{2 s} \leq s_{2}$. Now, let

$$
k=\left(E+L_{s}-L_{1}\right)^{-1} .
$$

Therefore, we obtain

$$
M^{-1}=(\underbrace{-k\left(L_{2 s}-s_{2}\right) k}_{\widehat{\eta}} \quad k) .
$$

And we have

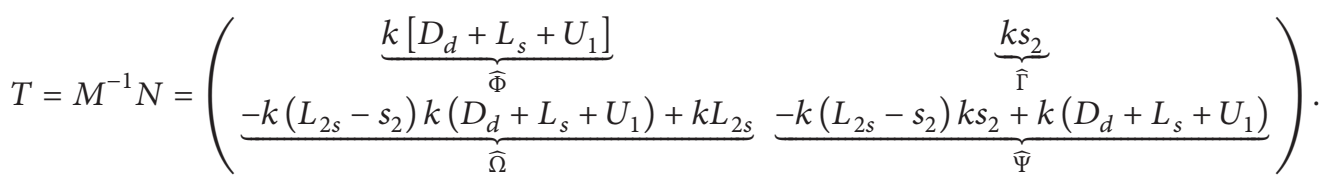

Now, from $X^{(i)}=\left[\frac{x^{(i)}}{\bar{x}^{(i)}}\right]$ we have the following algorithms (mentioned in [12] for the first time).

Algorithm 10. Mixed type splitting iterative methods_1 for FLS.

Step 1. Choose an initial vector $X^{(0)}=\left[\frac{x^{(0)}}{\bar{x}^{(0)}}\right]$ and parameters $r$ and $w$.

Step 2. For $k=0,1,2, \ldots$ do

$$
\begin{gathered}
\underline{x}^{(i+1)}=\widehat{\Phi} \underline{x}^{(i)}+\widehat{\Gamma} \bar{x}^{(i)}+(k \underline{b}) \\
\bar{x}^{(i+1)}=\widehat{\Psi} \bar{x}^{(i)}+\widehat{\Omega} \underline{x}^{(i)}+(\widehat{\eta} \underline{b}+k \bar{b}) .
\end{gathered}
$$

Step 3. If $\left\|\bar{x}^{(i+1)}-\bar{x}^{(i)}\right\| /\left\|\bar{x}^{(i+1)}\right\|<\varepsilon$ and $\left\|\underline{x}^{(i+1)}-\underline{x}^{(i)}\right\| /\left\|\underline{x}^{(i+1)}\right\|<$ $\varepsilon$, then stop; otherwise set $k=k+1$ and go to Step 2 .

Step 4. End for.

Step 5. Return to $X^{(k+1)}$.

Note that when

$$
D_{d}=\frac{1}{w}(1-w) D_{1}, \quad L_{s}=0, \quad L_{2 s}=0,
$$

we have SOR method for FLS (see [11]) and when

$$
D_{d}=\frac{1}{w}(1-w) D_{1}, \quad L_{s}=\frac{1}{w}(w-r) L_{1}, \quad L_{2 s}=0,
$$

where $(w, r)$ are real parameters with $w \neq 0$, we have AOR method for FLS (see [12]).

Theorem 11. Let $A$ be an H-matrix and let conditions of Lemma 7 be satisfied.

Then we have

$$
\begin{aligned}
& \text { (i): } \rho(T) \leq \rho(\langle T\rangle) \leq \rho\left(\left\langle l_{r, w}\right\rangle\right)<1, \\
& \text { (ii): } \rho(T) \leq \rho(\langle T\rangle) \leq \rho\left(\left\langle l_{w}\right\rangle\right)<1 .
\end{aligned}
$$

Proof. We only prove (i); (ii) can be similarly verified.

Let $A$ be an $H$-matrix. Then $\langle A\rangle$ is an $M$-matrix and by Lemmas 5 and 7 ,

$$
\rho(\langle T\rangle) \leq \rho\left(\left\langle l_{r, w}\right\rangle\right)<1 .
$$

By definition of mixed splitting method, we have

$$
\begin{aligned}
|T|=\mid & \left|\left(D+D_{d}+L_{l}-L\right)^{-1}\left(D_{d}+L_{l}+U\right)\right| \\
=\mid & \mid E\left(I-E^{-1}\left(L-L_{l}\right)\right)^{-1} \\
& \times\left[E\left\{E^{-1} D_{d}+E^{-1} L_{l}+E^{-1} U\right\}\right] \mid
\end{aligned}
$$




$$
\begin{aligned}
= & \left|\left(I-E^{-1}\left(L-L_{l}\right)\right)^{-1}\left[E^{-1} D_{d}+E^{-1} L_{l}+E^{-1} U\right]\right| \\
= & \mid\left(I+\left(E^{-1}\left(L-L_{l}\right)\right)\right. \\
& \left.+\left(E^{-1}\left(L-L_{l}\right)\right)^{2}+\cdots\right)\left[E^{-1} D_{d}+E^{-1} L_{l}+E^{-1} U\right] \mid \\
= & \mid\left(\left(E^{-1}\left(L-L_{l}\right)\right)+\left(E^{-1}\left(L-L_{l}\right)\right)^{2}+\cdots\right) \\
& \times\left[E^{-1} D_{d}+E^{-1} L_{l}+E^{-1} U\right] \\
& +\left[E^{-1} D_{d}+E^{-1} L_{l}+E^{-1} U\right] \mid \\
\leq & \left(\left\{\left|E^{-1}\left(L-L_{l}\right)\right|+\left(\left|E^{-1}\left(L-L_{l}\right)\right|\right)^{2}+\cdots\right\}\right. \\
& \left.\quad \times\left[\left|E^{-1} D_{d}\right|+\left|E^{-1} L_{l}\right|+\left|E^{-1} U\right|\right]\right) \\
& +\left(\left[\left|E^{-1} D_{d}\right|+\left|E^{-1} L_{l}\right|+\left|E^{-1} U\right|\right]\right) \\
= & \left(I-\left|E^{-1}\left(L-L_{l}\right)\right|\right)^{-1}\left(\left|E^{-1} D_{d}\right|+\left|E^{-1} L_{l}\right|+\left|E^{-1} U\right|\right) \\
& \\
& \\
= & (|T|) \leq \rho(\langle T\rangle) .
\end{aligned}
$$

Therefore we have

$$
\rho(T) \leq \rho(|T|) \leq \rho(\langle T\rangle)
$$

And the proof is completed.

Lemma 12. The Matrix $S$ in (5) or (6) is an H-matrix if and only if $A$ in (1) is H-matrix.

Proof. Let $A$ be an $H$-matrix; then by Lemma 8 , there exists a positive diagonal matrix $W$ such that $A W$ is strictly diagonally dominant matrix. Without loss of generality, let $A W$ be row strictly diagonally dominant; that is,

$$
\sum_{\substack{j=1 \\ j \neq i}}^{n}\left|a_{i j} w_{j j}\right|<\left|a_{i i} w_{i i}\right|
$$

Now, let

$$
j^{+}=\left\{a_{i j} \mid a_{i j} \geq 0\right\}, \quad j^{-}=\left\{a_{i j} \mid a_{i j}<0\right\}, \quad 1 \leq i, j \leq n ;
$$

then we have

$$
\sum_{\substack{j=1 \\ j \neq i}}^{n}\left|a_{i j} w_{j j}\right|=\sum_{\substack{j \in j^{+} \\ j \neq i}}\left|a_{i j} w_{j j}\right|+\sum_{\substack{j \in j^{-} \\ j \neq i}}\left|a_{i j} w_{j j}\right|<\left|a_{i i} w_{i i}\right| .
$$

By considering the structure of $S$ and since, for all $i=1, \ldots, n$, $a_{i i} \geq 0$, we have

$$
\begin{array}{r}
\sum_{\substack{j=1 \\
j \neq i}}^{n}\left|s_{i j}\right|=\sum_{\substack{j \in j^{+} \\
j \neq i}}\left|a_{i j}\right|, \quad \sum_{j=n+1}^{2 n}\left|s_{i j}\right|=\sum_{j \in j^{-}}\left|a_{i j}\right|, \\
1 \leq i \leq n, \\
\sum_{j=1}^{n}\left|s_{i j}\right|=\sum_{j \in j^{-}}\left|a_{i j}\right|, \quad \sum_{\substack{j=n+1 \\
j \neq i}}^{2 n}\left|s_{i j}\right|=\sum_{\substack{j \in j^{+} \\
j \neq i}}\left|a_{i j}\right|, \\
n+1 \leq i \leq 2 n .
\end{array}
$$

Therefore

$$
\begin{gathered}
\sum_{\substack{j=1 \\
j \neq i}}^{n}\left|s_{i j} w_{j j}\right|=\sum_{\substack{j \in j^{+} \\
j \neq i}}\left|a_{i j} w_{j j}\right|+\sum_{\substack{j \in j^{-} \\
j \neq i}}\left|a_{i j} w_{j j}\right|<\left|a_{i i} w_{i i}\right| \\
1 \leq i \leq n, \\
\sum_{\substack{j=1 \\
j \neq i}}^{n}\left|s_{i j} w_{j j}\right|=\sum_{\substack{j \in j^{-} \\
j \neq i}}\left|a_{i j} w_{j j}\right|+\sum_{\substack{j \in j^{+} \\
j \neq i}}\left|a_{i j} w_{j j}\right|<\left|a_{i i} w_{i i}\right| \\
\Longrightarrow \sum_{\substack{j=1 \\
j \neq i}}^{2 n}\left|s_{i j} w_{j j}\right|<\left|a_{i i} w_{i i}\right|=\left|s_{i i} w_{i i}\right| .
\end{gathered}
$$

Then, by choice of

$$
T=\left(\begin{array}{cc}
W & 0 \\
0 & W
\end{array}\right)_{2 n \times 2 n}
$$

ST is row SDDM. Therefore, by Lemma $8, S$ is also an $H$ matrix. Conversely, if $S$ is an $H$-matrix, then by reasoning similar to that above, it can be seen that $A$ is an $H$-matrix too.

Therefore, we can obtain the following theorem.

Theorem 13. Let $A$ be an H-matrix and the following conditions are satisfied:

$$
\begin{gathered}
0 \leq D_{d} \leq \frac{1}{w}(1-w) D_{1}, \quad 0 \leq L_{l} \leq \frac{1}{w}(w-r) L_{1}, \\
0 \leq L_{2 l} \leq s_{2} .
\end{gathered}
$$

Then the speed of convergence of Algorithm 9 is faster than the speed of convergence of SOR and AOR methods for FLS.

Proof. Let $A$ be an $H$-matrix; then by Lemma $12, S$ is an $H$-matrix too. Furthermore, by Theorem 11, $\rho\left(\left\langle l_{r, w}\right\rangle\right)<1$. Moreover, similar to the proving process of Theorem 11 and (31), we can obtain

$$
\rho\left(l_{r, w}\right) \leq \rho\left(\left|l_{r, w}\right|\right) \leq \rho\left(\left\langle l_{r, w}\right\rangle\right) .
$$


TABLE 1: $\left(D_{d}=0.1(-1+1 / w) D_{1} ; L_{s}=0.5(1-r / w) L_{1} ; L_{2 s}=0.01 s_{2}\right)$.

\begin{tabular}{|c|c|c|c|c|c|c|c|c|c|c|c|}
\hline \multicolumn{3}{|c|}{ Method } & \multicolumn{3}{|c|}{ AOR or SOR iterative methods } & \multicolumn{3}{|c|}{ Algorithm 10} & \multicolumn{3}{|c|}{ Algorithm 14} \\
\hline$n$ & $w$ & $r$ & Iter. & ELP & $\rho$ & Iter. & ELP & $\rho$ & Iter. & ELP & $\rho$ \\
\hline \multirow{4}{*}{5} & 0.5 & 0.1 & 64 & 0.003005 & 0.6739 & 20 & 0.001933 & 0.3764 & 20 & 0.001228 & 0.3228 \\
\hline & 0.5 & 0.5 & 62 & 0.003032 & 0.6322 & 17 & 0.001876 & 0.2278 & 15 & 0.001183 & 0.1476 \\
\hline & 0.7 & 0.3 & 34 & 0.001592 & 0.5191 & 16 & 0.001545 & 0.2530 & 16 & 0.001199 & 0.1953 \\
\hline & 0.9 & 0.6 & 21 & 0.001523 & 0.3192 & 13 & 0.001109 & 0.1399 & 12 & 0.001056 & 0.1084 \\
\hline \multirow{4}{*}{50} & 0.5 & 0.1 & 528 & 0.034716 & 0.9173 & 219 & 0.024478 & 0.9261 & 188 & 0.019920 & 0.5229 \\
\hline & 0.5 & 0.5 & 460 & 0.032090 & 0.8419 & 142 & 0.020052 & 0.8847 & 126 & 0.015032 & 0.6006 \\
\hline & 0.7 & 0.3 & 359 & 0.029076 & 0.8241 & 175 & 0.019944 & 0.9072 & 152 & 0.018738 & 0.4894 \\
\hline & 0.9 & 0.6 & 189 & 0.019587 & 0.9227 & 140 & 0.015078 & 0.8831 & 125 & 0.013490 & 0.6094 \\
\hline \multirow{4}{*}{100} & 0.5 & 0.1 & 1123 & 0.069947 & 0.9290 & 403 & 0.053815 & 0.9611 & 343 & 0.069539 & 0.5275 \\
\hline & 0.5 & 0.5 & 652 & 0.060993 & 0.8534 & 264 & 0.041211 & 0.9392 & 233 & 0.060378 & 0.6449 \\
\hline & 0.7 & 0.3 & 753 & 0.057825 & 0.8544 & 324 & 0.046366 & 0.9511 & 279 & 0.053829 & 0.5134 \\
\hline & 0.9 & 0.6 & 452 & 0.051150 & 0.6884 & 260 & 0.037807 & 0.9383 & 232 & 0.053185 & 0.6554 \\
\hline \multirow{4}{*}{250} & 0.5 & 0.1 & 2578 & 3.042835 & 0.9672 & 920 & 0.898741 & 0.9840 & 780 & 0.795855 & 0.5320 \\
\hline & 0.5 & 0.5 & 1993 & 1.967367 & 0.9302 & 619 & 0.635806 & 0.9749 & 549 & 0.582322 & 0.6738 \\
\hline & 0.7 & 0.3 & 1553 & 1.566464 & 0.9419 & 746 & 0.761868 & 0.9798 & 644 & 0.656939 & 0.5342 \\
\hline & 0.9 & 0.6 & 882 & 0.885911 & 0.8819 & 607 & 0.617928 & 0.9745 & 543 & 0.571853 & 0.6854 \\
\hline
\end{tabular}

Therefore,

$$
\rho\left(l_{r, w}\right)<1
$$

and by Lemma 7 the proof is completed.

In the sequel, we will improve Algorithm 10 by the following updating technique.

(I) First, use $\underline{x}^{i}$ and $\bar{x}^{i}$ from our previous information to compute the new $\underline{x}^{(i+1)}$ exactly as in the first line of Step 2.

(II) Then, replace $\underline{x}^{(i)}$ in the second line of Step 2 with $\underline{x}^{(i+1)}$, obtained just as in the above, to compute the new $\bar{x}^{(i+1)}$.

Algorithm 14. Mixed type splitting iterative methods_2 for FLS.

Step 1. Choose an initial vector $X^{(0)}=\left[\frac{x^{(0)}}{\bar{x}^{(0)}}\right]$ and parameters $r$ and $w$

Step 2. For $k=0,1,2, \ldots$ do

$$
\begin{gathered}
\underline{x}^{(i+1)}=\widehat{\Phi} \underline{x}^{(i)}+\widehat{\Gamma} \bar{x}^{(i)}+(k \underline{b}), \\
u p d=\widehat{\Omega} \underline{x}^{(i+1)} \\
\bar{x}^{(i+1)}=\widehat{\Psi} \bar{x}^{(i)}+u p d+(\widehat{\eta} \underline{b}+k \bar{b}) .
\end{gathered}
$$

Step 3. If $\left\|\bar{x}^{(i+1)}-\bar{x}^{(i)}\right\| /\left\|\bar{x}^{(i+1)}\right\|<\varepsilon$ or $\left\|\underline{x}^{(i+1)}-\underline{x}^{(i)}\right\| /\left\|\underline{x}^{(i+1)}\right\|<$ $\varepsilon$, then stop; otherwise set $k=k+1$ and go to Step 2 .

Step 4. End for.

Step 5. Return to $X^{(k+1)}$.

\section{Numerical Example}

In this section, we give an example of FLS to illustrate the results obtained in previous sections.

Example 1. Consider the $n \times n$ fuzzy system

$$
\begin{gathered}
2 x_{1}-x_{3}=(2+r, 4-r), \\
x_{2}+2 x_{3}-x_{4}=(2+r, 4-r), \\
x_{3}+2 x_{4}-x_{5}=(2+r, 4-r),
\end{gathered}
$$

$$
\begin{gathered}
x_{n-3}+2 x_{n-2}=(2+r, 4-r), \\
x_{n-2}+2 x_{n-1}=(2+r, 4-r), \\
x_{n-1}+2 x_{n}-x_{1}=(2+r, 4-r) .
\end{gathered}
$$

The extended $2 n \times 2 n$ matrix is

$$
S=\left[\begin{array}{lr}
s_{1} & -s_{2} \\
-s_{2} & s_{1}
\end{array}\right]
$$


where

$$
\begin{gathered}
s_{1}=\left[\begin{array}{ccccccc}
2 & 0 & \cdots & 0 & 0 & 0 & 0 \\
1 & 2 & \cdots & 0 & 0 & 0 & 0 \\
\vdots & \ddots & \ddots & \cdots & \ddots & \ddots & \vdots \\
0 & \cdots & 1 & 2 & 0 & \cdots & 0 \\
0 & \cdots & 0 & 1 & 2 & 0 & 0 \\
0 & \cdots & \ddots & 0 & 1 & 2 & 0 \\
0 & \cdots & \cdots & 0 & 0 & 1 & 2
\end{array}\right], \\
s_{2}=\left[\begin{array}{ccccccc}
0 & 0 & 0 & -1 & 0 & \cdots & 0 \\
0 & 0 & 0 & 0 & -1 & \ddots & \vdots \\
\vdots & \vdots & \ddots & \ddots & \ddots & \ddots & 0 \\
0 & \cdots & \cdots & 0 & 0 & \ddots & -1 \\
0 & \cdots & \cdots & 0 & 0 & 0 & 0 \\
0 & \cdots & \cdots & 0 & 0 & 0 & 0 \\
-1 & 0 & \cdots & 0 & 0 & 0 & 0
\end{array}\right] .
\end{gathered}
$$

Evidently, $A$ is an $H$-matrix and therefore $S$ is also an $H$ matrix. Table 1 shows the numerical results of the above example with the tolerance $\varepsilon=10^{-10}$. Moreover, the initial approximation is zero vector. In the Table 1, we reported the number of iterations (Iter), Elapsed time (ELP), and associated spectral radii $(\rho)$ for the iterative methods with different $n, w$, and $r$. This results show that our algorithms can be applied to a large class of FLS. Furthermore, from Table 1, we can see that for solving FLS, Algorithm 14 is superior to the other methods from point of view rate of convergence.

This example is computed with MATLAB7 on a personal computer Pentium 4-256 MHZ.

\section{Conclusion}

In this paper, we have proposed a class of splitting methods, called mixed type splitting iterative method for fuzzy linear systems that contains auxiliary matrices. Furthermore, our method with some other iterative methods is compared in the frame of fuzzy linear systems and it is shown that by proper choice of these auxiliary matrices, the new iterative method is faster than these mentioned methods form point of view of the convergence speed.

\section{Conflict of Interests}

The authors declare that there is no conflict of interests regarding the publication of this paper.

\section{References}

[1] A. Kandel, M. Friedman, and M. Ma, "Fuzzy linear systems and their solution," in Proceedings of the IEEE International Conference on Systems, Man and Cybernetics, vol. 1, pp. 336-338, October 1996.

[2] M. Friedman, M. Ming, and A. Kandel, "Fuzzy linear systems," Fuzzy Sets and Systems, vol. 96, no. 2, pp. 201-209, 1998.

[3] M. Friedman, M. Ma, and A. Kandel, "Duality in fuzzy linear systems," Fuzzy Sets and Systems, vol. 109, no. 1, pp. 55-58, 2000.
[4] J. J. Buckley and Y. Qu, "Solving fuzzy equations: a new solution concept," Fuzzy Sets and Systems, vol. 39, no. 3, pp. 291-301, 1991.

[5] L. A. Zadeh, "Fuzzy sets," Information and Computation, vol. 8, pp. 338-353, 1965.

[6] H. Saberi Najafi and S. A. Edalatpanad, "On the Nash equilibrium solution of fuzzy bimatrix games," International Journal of Fuzzy Systems and Rough Systems, vol. 5, no. 2, pp. 93-97, 2012.

[7] S. A. Edalatpanah and S. Shahabi, "A new two-phase method for the fuzzy primal simplex algorithm," International Review of Pure and Applied Mathematics, vol. 8, no. 2, pp. 157-164, 2012.

[8] H. S. Najafi and S. A. Edalatpanah, "A note on "a new method for solving fully fuzzy linear programming problems"', Applied Mathematical Modelling, vol. 37, no. 14-15, pp. 7865-7867, 2013.

[9] E. Abdolmaleki and S. A. Edalatpanah, "Fast iterative method (FIM) for solving fully fuzzy linear systems," Information Sciences and Computing, no. 1, Article ID ISC050713, 9 pages, 2013.

[10] T. Allahviranloo, "Numerical methods for fuzzy system of linear equations," Applied Mathematics and Computation, vol. 155, no. 2, pp. 493-502, 2004.

[11] T. Allahviranloo, "Successive over relaxation iterative method for fuzzy system of linear equations," Applied Mathematics and Computation, vol. 162, no. 1, pp. 189-196, 2005.

[12] M. Dehghan and B. Hashemi, "Iterative solution of fuzzy linear systems," Applied Mathematics and Computation, vol. 175, no. 1, pp. 645-674, 2006.

[13] S. Miao, B. Zheng, and K. Wang, "Block SOR methods for fuzzy linear systems," Journal of Applied Mathematics and Computing, vol. 26, no. 1-2, pp. 201-218, 2008.

[14] H. Saberi Najafi and S. A. Edalatpanah, "Preconditioning strategy to solve fuzzy linear systems (FLS)," International Review of Fuzzy Mathematics, vol. 7, no. 2, pp. 65-80, 2012.

[15] H. S. Najafi and S. A. Edalatpanah, "An improved model for iterative algorithms in fuzzy linear systems," Computational Mathematics and Modeling, vol. 24, no. 3, pp. 443-451, 2013.

[16] H. Saberi Najafi, S. A. Edalatpanah, and A. H. Refahi Sheikhani, "Application of homotopy perturbation method for fuzzy linear systems and comparison with Adomian's decomposition method," Chinese Journal of Mathematics, vol. 2013, Article ID 584240, 7 pages, 2013.

[17] C. Li, X. Liang, and D. J. Evans, "An iterative method for the positive real linear system," International Journal of Computer Mathematics, vol. 78, no. 1, pp. 153-163, 2001.

[18] C. Li and D. J. Evans, "Note to the mixed-type splitting iterative method for the positive real linear system," International Journal of Computer Mathematics, vol. 79, no. 11, pp. 1201-1209, 2002.

[19] G.-H. Cheng, T.-Z. Huang, and S.-Q. Shen, "Note to the mixedtype splitting iterative method for $Z$-matrices linear systems," Journal of Computational and Applied Mathematics, vol. 220, no. 1-2, pp. 1-7, 2008.

[20] A. Berman and R. J. Plemmons, Nonnegative Matrices in the Mathematical Sciences, Academic Press, New York, NY, USA, 1994. 

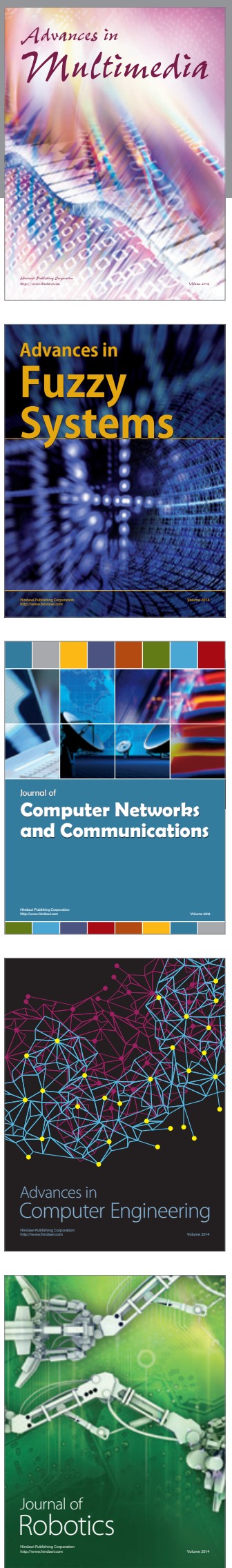

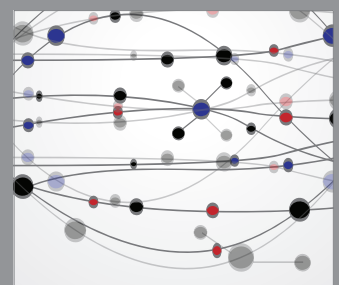

The Scientific World Journal
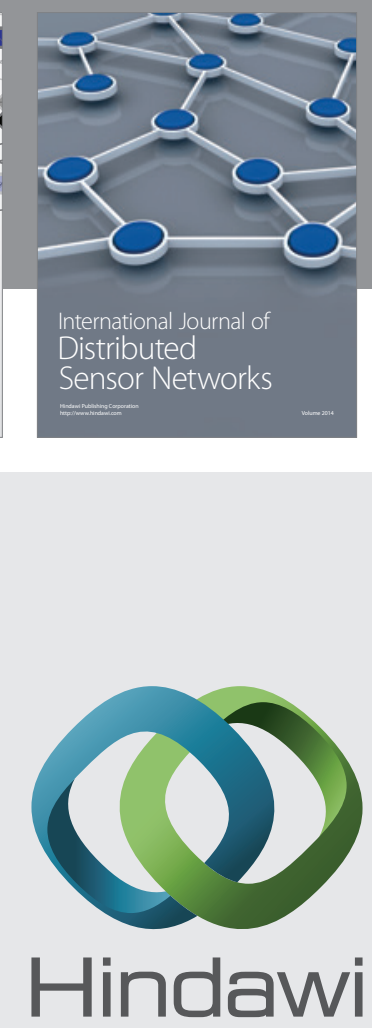

Submit your manuscripts at

http://www.hindawi.com
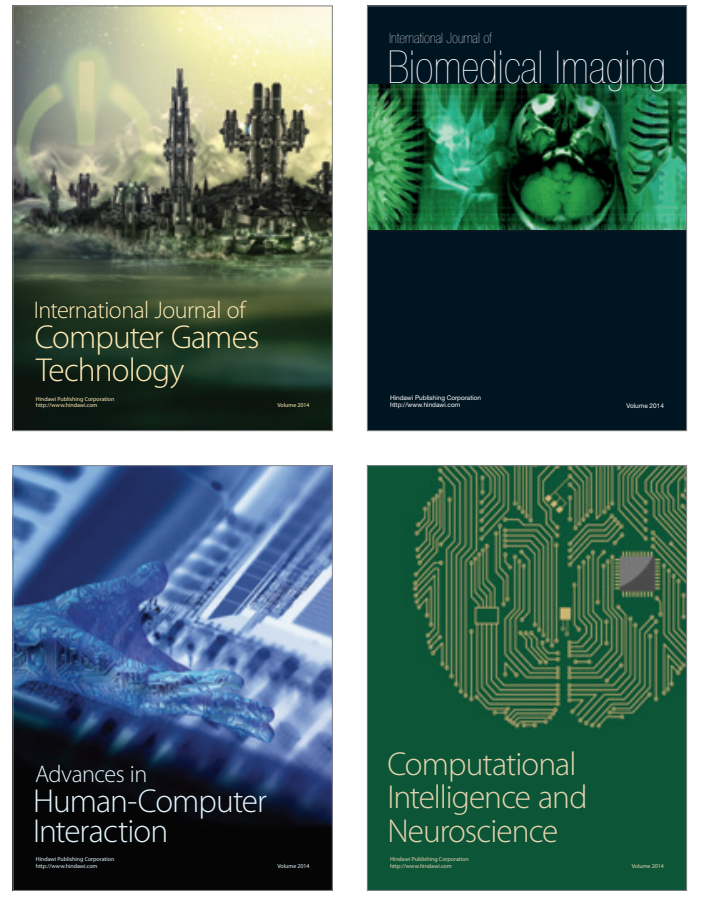
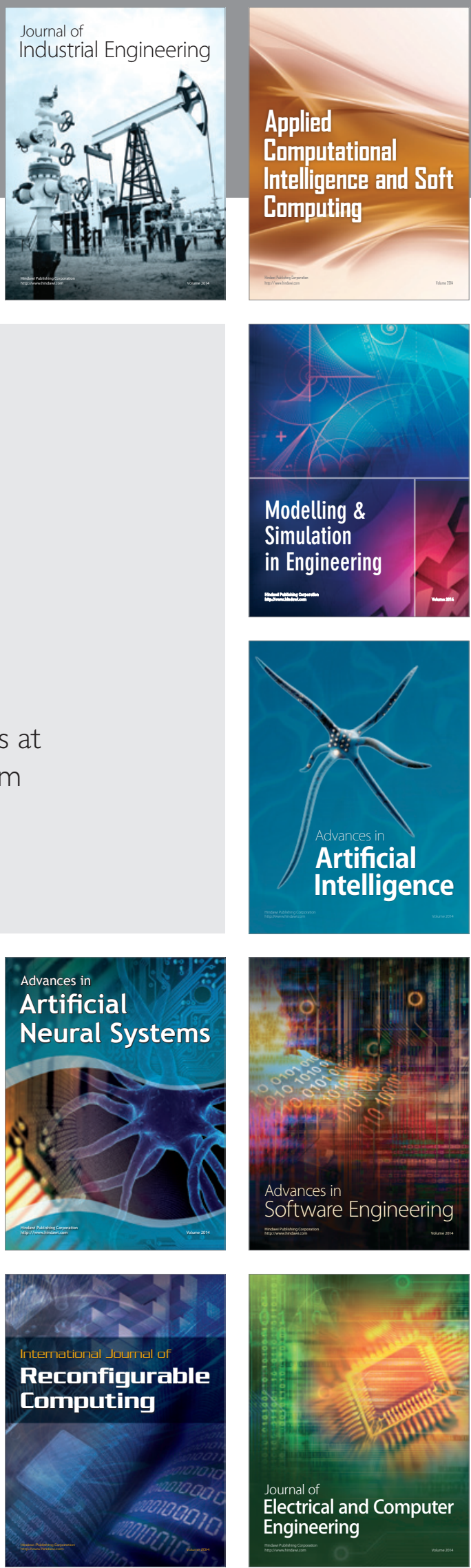